\title{
THE IMPACT OF EDUCATION ON BREAST CANCER SURVIVAL IN THE STATE OF SÃO PAULO
}

Raissa Janine de Almeida1, Carolina Terra de Moraes Luizaga2, José Eluf-Neto2,3, Eduardo Carvalho Pessoa1, Amanda de Moraes Mamede Chiarotti1, Rainer de Almeida Souza1, Cristiane Murta-Nascimento1

1Universidade Estadual Paulista "Júlio de Mesquita Filho", Medical School - Botucatu (SP), Brazil;

2Fundação Oncocentro de São Paulo - São Paulo (SP), Brazil.

3Faculty of Medicine, Universidade de São Paulo - São Paulo (SP), Brazil

Objectives: To estimate 5- and 10-year breast cancer-specific survival probabilities of patients admitted in the hospitalbased cancer registry (HBCR) of the Fundação Oncocentro de São Paulo (FOSP, in Portuguese) and to assess the prognostic factors for this neoplasm. Methods: Historical cohort study that included women with breast cancer included in HBCRFOSP and diagnosed between 2002 and 2012. The event of interest was breast cancer-specific mortality. Living cases at the end of follow-up (December 31, 2017), loss to follow-up, and death other than that due to breast cancer were considered censored on the date of the last contact or date of death. Descriptive analysis and survival analysis were performed using the Kaplan-Meyer method. Survival curves were compared using the log-rank test. HR and 95\%CI were estimated using Cox proportional hazards model. This study was approved by the Human Research Ethics Committee of the Botucatu Medical School, São Paulo State University, Brazil. Results: The HBCR-FOSP registered 53,146 cases of invasive breast cancer between 2002 and 2012. The median age at diagnosis was 55.9 years. The 5- and 10-year breast cancer-specific survival for the entire cohort was $76.1 \%$ (95\% CI 75.7-76.5) and 64.8\% (95\%CI 64.2-65.3), respectively. In the multivariate analysis, the factors clinical stage and educational level were with the greatest impact on survival. The other factors associated with prognosis were age at diagnosis, histological type, and year of diagnosis. Conclusion: The results show that patients in more advanced stages and with less level of education have a higher risk of death from breast cancer. Besides, these findings may contribute to the development of policies for the identification of breast tumors at earlier stages.

Keywords: Breast Neoplasms; Survival Analysis; Prognosis. 